\title{
Freeform Command and Control of Robotic Platforms
}

\author{
Paul Scerri ${ }^{1}$ \\ Robotics Institute, Carnegie Mellon University, Pittsburgh, PA 15213, USA \\ David Van Brackle ${ }^{2}$ \\ Lockheed Martin, Advanced Technology Laboratories, Kennesaw, G, 30144, USA \\ Christopher Rouff ${ }^{3}$ \\ Lockheed Martin, Advanced Technology Laboratories, Arlington, V, 22203, USA \\ and \\ Harold Dick, Kevin Vallandingham ${ }^{4}$ \\ Lockheed Martin, Advanced Technology Laboratories, Leavenworth, KS 66048, USA
}

This is the abstract.

\section{Introduction}

\begin{abstract}
A $\mathrm{S}$ has been seen from the results of the DARPA Urban Challenge, autonomous robotic systems are becoming more robust and capable of executing complex missions over long durations of time. It is conceivable that within a few short years that there will be a large number of robotic systems available to assist the warfighter. In addition, the government has mandated that one-third of the military's vehicles be autonomous by 2015 , so there is strong backing to increase the number of unmanned systems on the battlefield. Integrating robotic vehicles into missions will require new methods to not only command the vehicles themselves but to integrate them into missions as equal members of a combat team.

Incorporating unmanned autonomous systems (UASs) into manned missions will be difficult. UASs, unlike humans, require precise commanding and do not tolerate ambiguity. There is still much work to be done in the robustness of UASs so mission planning will still need to plan for contingencies. Also, operating UASs currently requires large amounts of operator training. What is needed on the battlefield is for any soldier to be able to quickly and easily provide a plan, or collaboratively generate a plan with one or more UASs as part of a human-UAS team, and then quickly execute that plan while being able to replan on the fly.

Carnegie Mellon University (CMU) and Lockheed Martin ATL are developing a new paradigm for command and control (C2) of teams of autonomous assets and humans, referred to as Freeform C2 (F2C2). This concept allows one or more warfighters across echelons (if necessary) to sketch plans in an intuitive, interactive way and then work iteratively with an intelligent interface to refine and flesh out the details of the plan with other warfighters and the unmanned systems. The plan would be executed flexibly by intelligent, autonomous coordination of both humans and UASs. The autonomous coordination will feed information back to the warfighters where it will be overlaid on the plan. The warfighers can then intuitively and naturally modify or update the plan as needed.
\end{abstract}

\section{Sketching for Planning of Courses of Action}

As shown in figure 1, sketching is a natural means for people to communicate when expressing ideas or generating a plan. It is unrestrictive, fast, naturally cooperative, requires no training other than in the symbols that are used, and easily modified or updated. A number of technologies have been developed for understanding sketches and is becoming a mature technology ${ }^{1-7}$. Building on this, autonomic ${ }^{8,9}$ course of action (COA) understanding has also been shown to be feasible where a commander sketches out COA symbols and the system

\footnotetext{
${ }^{1}$ Senior Systems Scientist, 5000 Forbes Avenue, Pittsburgh, PA 15213.

${ }^{2}$ Senior Engineer, ISX Lab, 3550 George Busbee Parkway, Suite 210, Kennesaw, GA 30144.

${ }^{3}$ Engineering Management, ISX Lab, 4301 Fairfax Drive, Suite 500, Arlington, VA, 22203. AIAA Member.

${ }^{4}$ Program Manager, ISX Lab, 2225 Vilas Street, Leavenworth, KS 66048.
} 


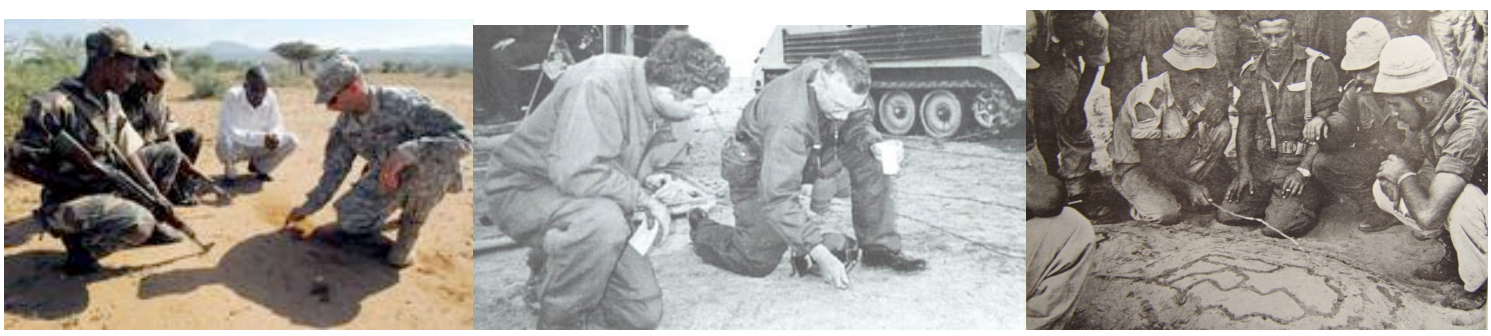

Figure 1. Freeform drawing is a natural way for people to describe courses of actions.

interprets the drawings and redraws them based on the inferred meaning (see Figure 2) ${ }^{10}$. Such COA inference can be used to collaborate with and produce UAS plans.

Sketching of plans and actions has also been done in RoboCup ${ }^{11,12}$ competitions. The RoboCup simulation competition pits teams of intelligent agents against each other in a game of soccer. Like military operations, a key component of a successful team is to design team strategies that are effective at beating the opponent's strategy. Tactic specification is time-consuming because there are so many situations that the team may find itself in, difficult because tactics for different situations must be individually effective and must fit well with other tactics that the team may transition to as the situation changes, and iterative because the first set of tactics specified are unlikely to be ideal. Critically, the developers that create the underlying artificial intelligence (AI) for the agents in the game are not the same experts that understand effective soccer tactics. To facilitate domain experts specifying team tactics, CMU developed a tool that allowed experts to specify tactics in a way that they are used to and comfortable with, namely by using a whiteboard sketching metaphor ${ }^{13}$.

Domain experts "draw" on an image of a soccer pitch. Using a mouse the user "draws" the locations of players, one type of arrow for passing and another for when a player should dribble. What each player should do with the ball, in each situation can be specified by selecting the situation from a dropdown menu at the top of the screen. Figure 3 shows a screenshot of a specific tactic being defined. Because the interface is of a form that users are comfortable with, domain experts can quickly become proficient at designing tactics. At a university open day where visitors were encouraged to play with the tool, users as young as 6 years old with only minimal training were able to create and iteratively improve, based on watching the team play, a RoboCup simulation team.

The technical key to the approach is that the specification on the whiteboard can be "compiled" into a set of layered behavior-based controllers, one for each of the agents. The specification on the whiteboard effectively provided information for selection of an overall plan and parameter values for the details. The set of plans and ability to parameterize was quite restrictive but broad enough for conventional soccer tactics. We anticipate that using an underlying plan "language" that is more general and powerful would allow more flexible specification on the whiteboard and could be applicable to the military domain.

Others have also looked at building end-user specification tools for describing team behavior. A few examples include Akin's Mission Lab ${ }^{14}$ which gives end users the ability to specify multi-robot behaviors. Lewis ${ }^{15,16}$ and Parasuraman ${ }^{17,18}$ have developed interfaces and approaches that have given run-time control over teams of robots to relatively inexperienced users.

\section{Outline of F2C2 Concept}

The $\mathrm{F} 2 \mathrm{C} 2$ concept is aimed at spatially and temporally limited tactical operations. It is designed specifically for teams where there are many autonomous assets working alongside people to achieve an important tactical objective. The focus is on quickly and collaboratively constructing plans for a heterogeneous team, monitoring the execution of the plan and reacting to circumstances in the environment. The $\mathrm{F} 2 \mathrm{C} 2$ concept is specifically aimed at real-time operations when several people are responsible for planning and commanding a mission. There will be three major components of an $\mathrm{F} 2 \mathrm{C} 2$ system:

1. the interfaces,

2. underlying intelligence, and

3. inter-platform communications.

The interfaces are what people will interact with and represent a new interaction paradigm. The underlying intelligence will manage routine tasks, provide input on human plans and manage sensor assets and incoming sensor data. The inter-platform communication keeps all interfaces and assets synchronized during the mission so plans 


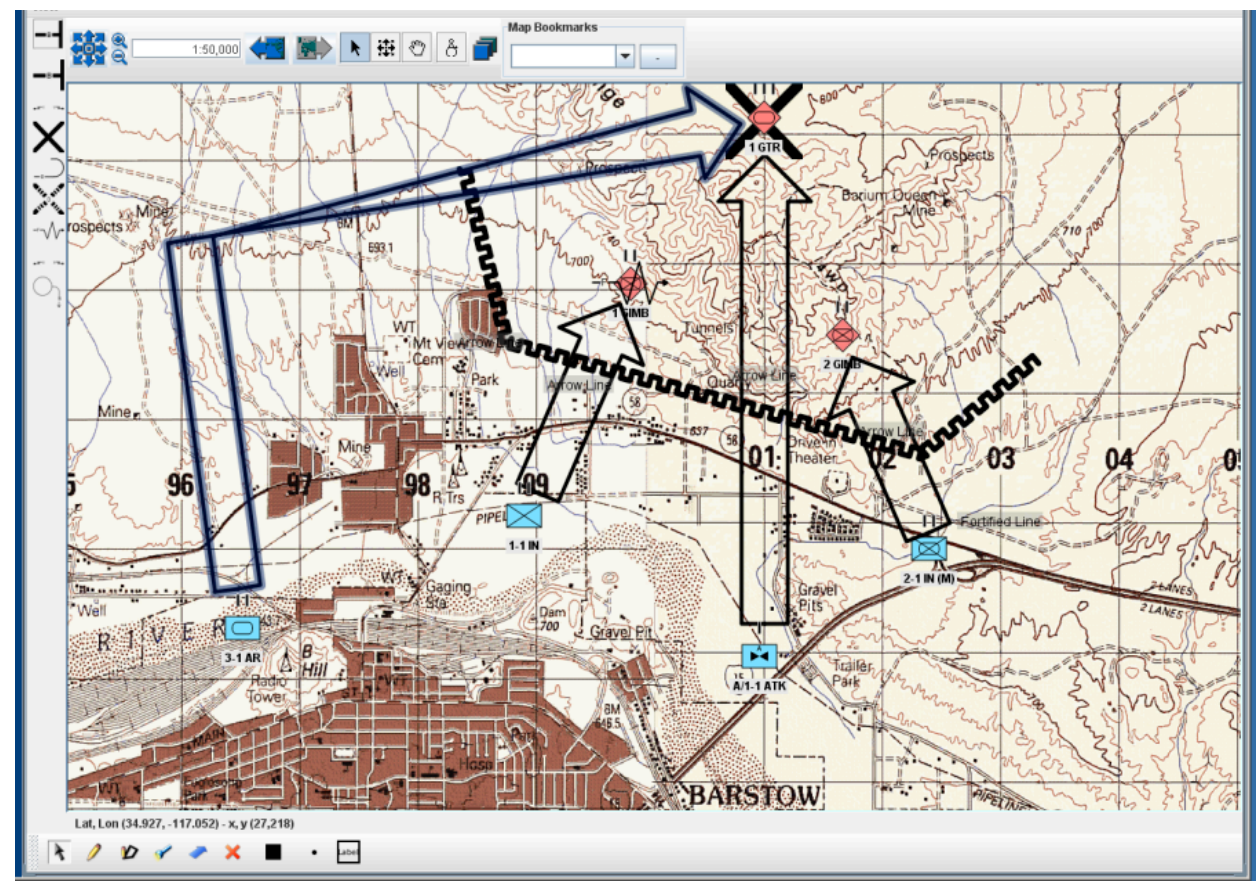

Figure 2. InCOA sketch-based system that performs automatic COA recognition. ${ }^{8}$

can be developed collaboratively and if there is a change to a mission all participants know about it immediately and can replan or take appropriate action.

To the people managing a mission, $\mathrm{F} 2 \mathrm{C} 2$ will appear as an interactive table that they interact with via touch. The table will display the current state of the planning and environment, with current sensor data directly integrated with the view of the environment. People will stand around the table and draw as they would normally on a map or whiteboard and be able to collaboratively develop plans with the people and machines in the field. The F2C2 system will interpret the drawings as plans, change free form drawings to corresponding icons or standard military COA symbols, fill in details, adding components where needed and possibly suggesting changes. Any of the people around the table (or in the field) can interact with the table, editing current plans or exploring options. The F2C2 system will continuously assist in refining, integrating and evaluating plans. Once mission execution begins, realtime data will update the table and people can modify, add to or improve their plans interactively or communicate with those executing the mission. Once again the $\mathrm{F} 2 \mathrm{C} 2$ system will assist in this process by managing details and suggesting options. When important events occur the F2C2 system will alert the people without preventing other activities from continuing.

A key philosophy in the concept is that cooperation between people and cooperation between people and computers is required to plan tactical missions and monitor and adjust to the changing circumstances. People need to cooperate because they bring different perspectives to the situation and because they have different background knowledge and current information. For example, a person planning UAV missions will have different information than a Commander with overall responsibility. The F2C2 concept acknowledges and encourages this cooperation by letting multiple people interact on the same interface, giving them the ability to not only see each other's actions, but communicate face-to-face about plans. Computers and people need to cooperate, because they have different strengths that should be used to complement one another for best overall performance. The F2C2 concept encourages this cooperation by using computer intelligence to interpret and flesh out plans specified by people. The computers should focus on detailed planning, being vigilant and processing large amounts of sensor data, leaving creative and knowledge intensive work to humans.

When people plan, especially cooperatively, they will sketch high-level ideas, then iteratively refine the plans, weaning out the ideas that turn out to be unworkable. The F2C2 concept encourages this planning process, while improving it by allowing computers to fill in details speeding the overall process. Mixed-initiative functionality will allow the system to proactively perform parts of the planning and suggest problems or alternatives when applicable. F2C2 will emphasize planning around teams and sub-teams meeting team level objectives, rather than planning the behavior of individual manned or unmanned assets. Where possible, planning the details of individual activities will 
be primarily performed by the system, with people only approving or iteratively improving computer designed plans. The F2C2 concept brings before action planning and during action adaptation into a combined framework and interface. An important consequence of this, apart from the obvious advantage of people needing to only understand the one interface, is that information generated during planning is automatically available to the system at runtime. For example, if a person had rejected a particular UAV flight path, for some reason, during initial planning, the system could avoid suggesting the same plan when the situation changed during execution.

Autonomous assets for data collection should not be the focus of a planning or execution process. The focus should only be on the data provided by the sensors. F2C2 abstracts away from sensing assets and simply renders the sensed data directly on the interface. When at all possible, the locations of the sensor assets will not be shown, allowing people to treat the interface as an up-to-date view of the world, without the additional overhead of managing a large number of sensor assets. The $\mathrm{F} 2 \mathrm{C} 2$ interfaces will need to be carefully designed to show both the data and allow access to the pedigree and age of the data displayed so that users of the information know what they are dealing with. To achieve this objective, autonomous planning for the sensing assets is required and must start with an autonomous interpretation of what data will be important. Mixed-initiative interaction will allow people to intervene with sensing plans when there are specific requirements or concerns. A key consequence of abstracting away from sensor details is that a single mission can support of the assets because humans are not required to manage them, thus providing the people with an even better view of the current situation.

While the primary planning and plan management for a tactical encounter will be done by people with the ability to use relatively large display media, useful input into the planning process may come from other people in the field without access to such media. For example, a special operations soldier on the ground may usefully edit planned convoy paths and suggest alternatives and potential locations of opponents. The $\mathrm{F} 2 \mathrm{C} 2$ concept is designed to be distributed across multiple physical interfaces and configurable to different display media. Configuration includes intelligently managing which data is shown on smaller media. This concept is key to the overall collaborative concept of free form command and control.

\section{System Overview}

Figure 4 gives a high level overview of the architecture for F2C2. The system is made up of hand-held or other sketch capable devices that are with the warfighters in the field, operations centers or at command headquarters. The devices are connected through a real-time collaboration framework that allows instant updating of collaborators

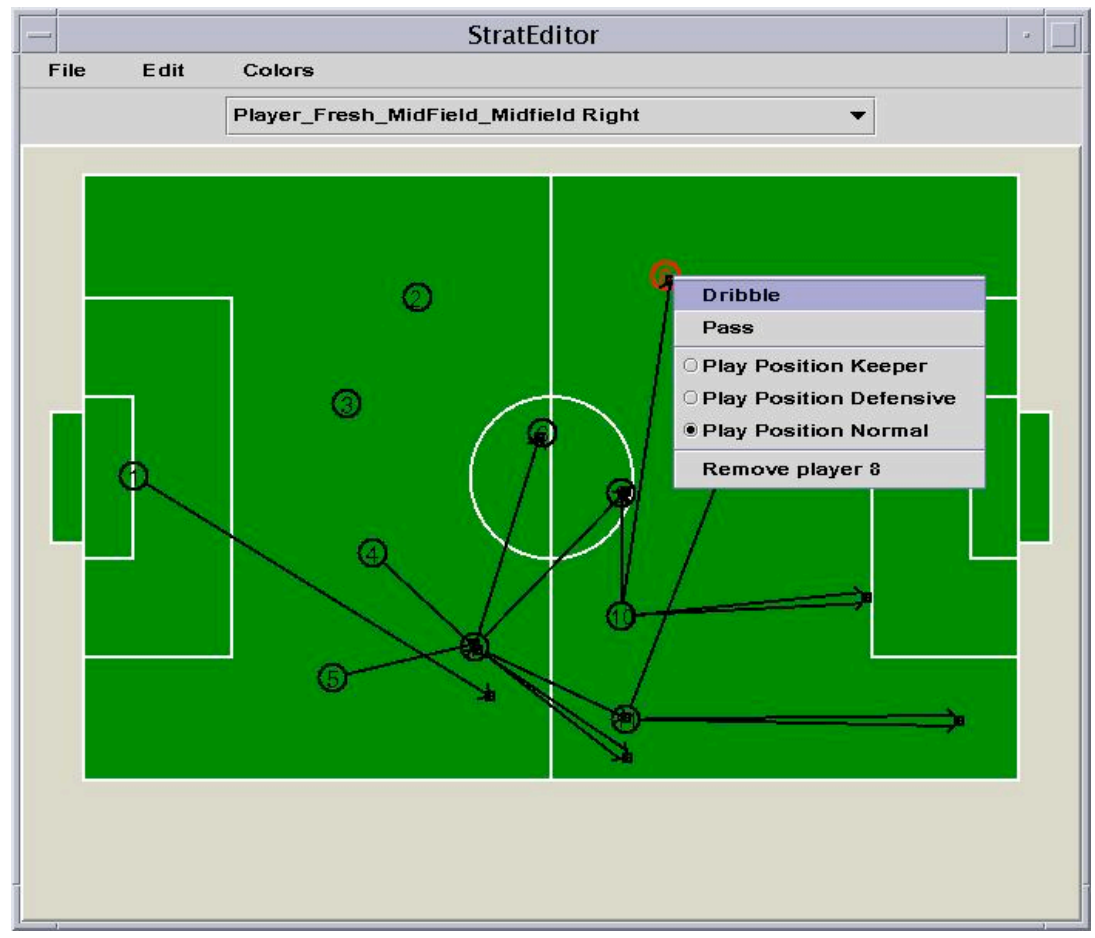

Figure 3. InCOA sketch-based system that performs automatic COA recognition. ${ }^{8}$

7

American Institute of Aeronautics and Astronautics 


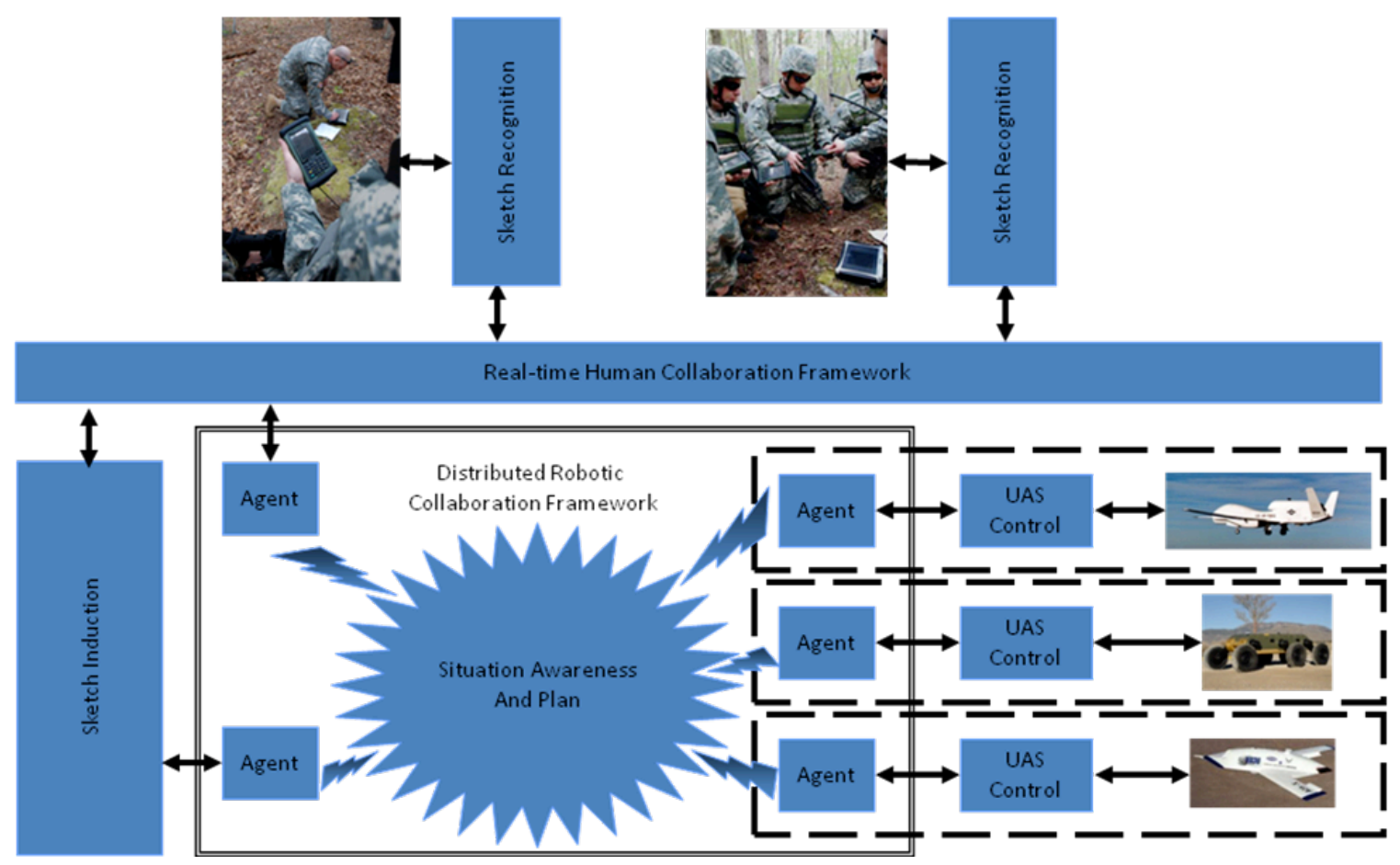

Figure 4. High level architecture of Freeform C2.

inputs as well as data coming from unmanned systems. This framework then provides the sketch induction of the drawings that are done by the warfighters. Icons and other military symbols are recognized and are converted on the users displays to the appropriate symbols. Agents for the warfighters and agents that act as proxies for the unmanned systems then collaborate on the plan as it is being formed. The following are the sets of technologies embedded in the framework to implement F2C2.

Intent inference is used to determine what the plan described by the sketch represents. This is in part gesture recognition, augmented with the use of standard military symbols, which is a mostly solved problem. More subtle is determining the details and implications of what the commander is trying to describe. For example, a straight line drawn through an area might represent an approximate path to be worked out in detail autonomously, while a more carefully drawn path might represent a specific route to follow. Much of this was implemented in InCOA (Figure $2)^{8}$.

Plan Elaboration is needed to fill out details in a plan when they are sketched out. When a commander describes a plan, many details may be assumed and would be filled in by human subordinates. For UASs, more detail needs to be provided. Feedback to the commanded can be used to confirm the commander's intent. For example, software might propose providing UAV oversight for a convoy. This requires understanding plans and finding efficient techniques for presenting options to the user.

Information Presentation is needed to display information coming from UASs, which may be uncertain, temporally varied and in a range of modalities.

Autonomous Coordination. To empower more UASs to work with fewer operators, UASs must autonomously coordination their activities so that operators can ignore the details of which UAS is doing what and focus on the information and actions produced by the UASs as a team.

Distributed Collaboration. Multiple distributed operators at different points in the command structure and in different physical environments need to work together to perform $\mathrm{C} 2$. As well as the collaboration challenges, different physical interfaces need to be used, from small tablets or HUDs for infantry in the field to large touch screen table-like displays that allow multiple operators to refine plans collaboratively.

\section{Scenario}

The following is a scenario of how $\mathrm{F} 2 \mathrm{C} 2$ would be used as a collaborative planning and execution tool between warfighters and unmanned system. The scenario is based on the 1-22 Infantry Battalion (IN BN) War-game where there was movement from a Tactical Assemble Area (TAA) to Assault Positions, and dynamic re-tasking based on

$$
7
$$

American Institute of Aeronautics and Astronautics 


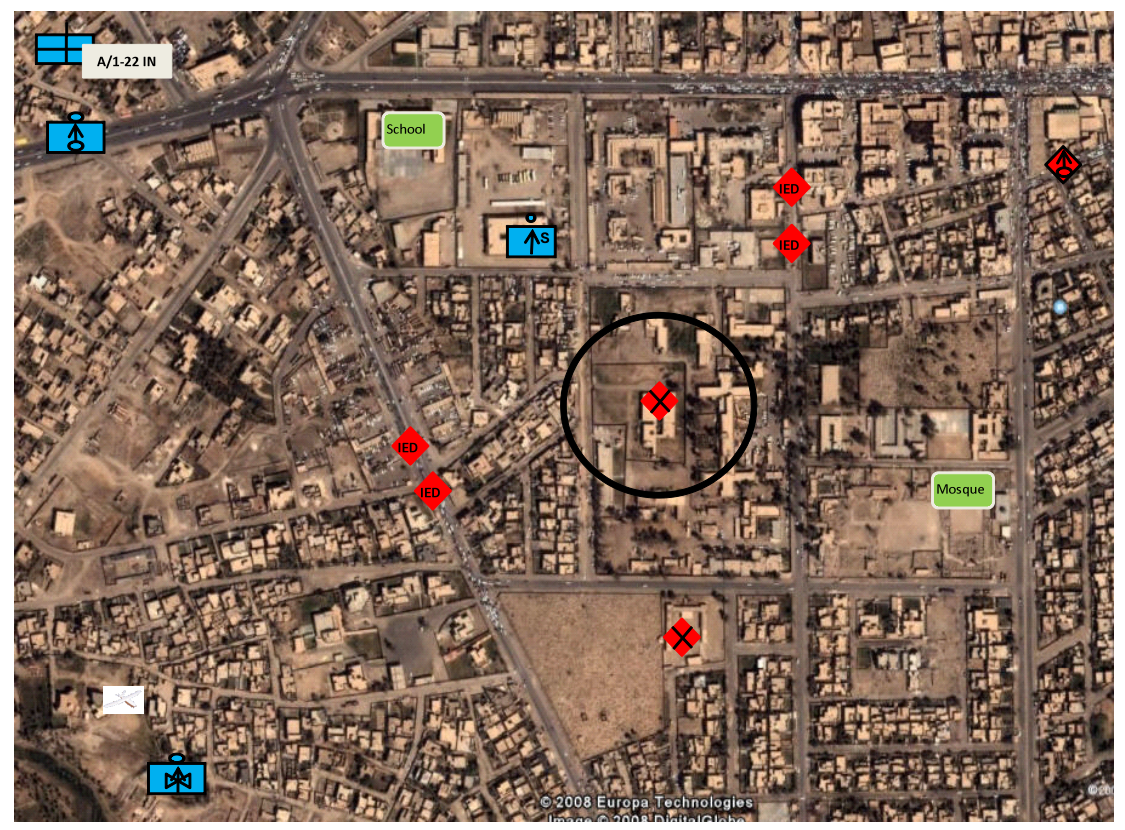

Figure 5. Operational graphics for scenario mission

updated Situation Reports (SITREPs). As the 1-22 BN staff prepared to conduct the war-game the BN S3 reviewed the task organization selected and the course of action directed by the commander. The selected COA had A/1-22 conducting a cordon and search to capture or kill Peridue. See Figure 5 for operational graphics.

Based on the commander's guidance and the initial array of forces the staff begins the wargame process. Using F2C2 the BN S2 (Intel), based on the war-game, annotates and enters the collection plan into the $\mathrm{F} 2 \mathrm{C} 2$ to assign collection assets to designated Named Areas of Interest (NAIs), Targeted Areas of Interest (TAIs), and Areas of Interest (AIs). The BN S3 Army Airspace Command and Control (A2C2) designates routes and deconflicts airspace.

Because $\mathrm{F} 2 \mathrm{C} 2$ is based on a deep collaborative system the end product is automatically shared with the assigned team from the Brigade Combat Team (BCT). At the end of the war game the UAV operators will know their assigned responsibilities (Task and Purpose), routes, and time lines.

During the war-game the BN S3 provides the following guidance for the UAV:

- Fly Route Blue (Ingress) to provide intelligence at NAI 001 where there have been 2 IED attacks in the past 48 hours. He states he want this accomplished 1 hour before A/1-22 IN departs the Assault position.

- Confirm or deny the presence of a SAPA platoon south of the OBJ area at NAI 002.

- Confirm or deny the mortar section NE of OBJ at TAI 001

- On order provide intelligence along Route Yellow (Egress Route) at NAI 003 where there have been 2 IED attacks in the past 48 hours.

As the $\mathrm{S} 2$ enters the areas for the UAV to gather information the $\mathrm{F} 2 \mathrm{C} 2$ automatically begins to do an assessment of the area to be observed. It understands line of sight and what altitude and distances are needed to maintain observation of the targeted area. In the past, a planner would simply assign and general $\mathrm{A} 2 \mathrm{C} 2$ measure in terms of the width and minimum and maximum elevations the UAV could operate with little or no actual analysis of the immediate terrain around the target (buildings are a great example). In Baghdad, structures are generally surrounded by high walls with a courtyard in the middle; buildings are very close and generally require varying altitudes to maintain visual observation of a targeted area. So an F2C2 enhanced UAV would understand the task given and the operating parameters (A2C2/threats) and will make necessary adjustments in speed, altitude, and 
recommend route changes, if it encounters an area that restricts its ability to provide observation (smoke, weather related instances).

Automatically the $\mathrm{F} 2 \mathrm{C} 2$ provides recommended routes based on task given, an analysis of possible threats (small arms fire/Air Defense fire/etc). It has also queried other F2C2 to deconflict the air space prior to giving the S2 recommendations. If the $\mathrm{S} 2$ has to alter the recommended routes it can be done by simply clicking the route and moving individual points to where they need to be. Even as the S2 is altering the routes the $\mathrm{F} 2 \mathrm{C} 2$ technology continues to conduct the analysis discussed above. If there is a possible threat on the S2s route it would shade that area red and show where the possible threat is located.

Within $\mathrm{F} 2 \mathrm{C} 2$ there is a system of prioritization determined by the Division G5 plans section. What this means is based on all of the operations taking place in the Division Air Operations (AO) they prioritize which systems have priority over others. This allows $\mathrm{F} 2 \mathrm{C} 2$ enhanced UAVs/UGVs to collaborate with each other to deconflict or recommend ways to deconflict airspace and ground-space. If a UAV is dynamically re-tasked it can automatically conduct an analysis of the airspace and threats and determine a route to get to the target. Of course the user can set restrictions on these decisions in order to intervene and add some art of war to what could be a pure science of war approach.

The $\mathrm{F} 2 \mathrm{C} 2$ also provides data specific to each route (time of flight for each displayed on a time line bar that allows the user to grab and drag along the timeline to show where the UAV position will be at a specific time). It also has a line of sight tool that allows the user to see what the UAV/UGV can or cannot see as it moves along its route. After the mission planning is done the operation is executed.

\section{Conclusion}

This paper presented a system concept called Free Form Command and Control (F2C2). In F2C2 unmanned systems and warfighters collaborate to develop and execute plans on the fly in the field. This technology is based on systems developed by Lockheed Martin Advanced Technology Laboratories and CMU that consists of a sketchbased course of action generation tool and a RoboCup tactical play specification tool. The motivation behind this work is the large number of unmanned and autonomous systems that are being developed. A warfighter controlling a large number of unmanned systems through the typical method of using a joystick while a executing a mission will not be possible. F2C2 strives to integrate unmanned systems as an equal member of a team without the overhead of current user control techniques.

\section{References} $-68$.

${ }^{1}$ Forbus, K., Usher, J., and Chapman, V. "Sketching for military courses of action diagrams," Proc IUI 03, ACM Press, pp. 61

${ }^{2}$ Kara, L. and Stahovich, T., "Hierarchical Parsing and Recognition of Hand-Sketched Diagrams," 17th ACM User Interface Software Technology (UIST) 2004.

${ }^{3}$ Sezgin, T. M. and Davis, R., "Sketch Interpretation Using Multiscale Models of Temporal Patterns,"ß27(1), pp.28-37. January 2007.

${ }^{4}$ Alvarado, C. and Davis, R., "SketchREAD: AMulti-Domain Sketch Recognition Engine," In Proceedings of UIST 2004, pp.23-32. New York, NY, October 24-27 2004.

${ }^{4}$ Sezgin, M.T., Stahovich, T., and Davis, R., "Sketch Based Interfaces: Early Processing for Sketch Understanding," InWorkshop on Perceptive User Interfaces, Orlando FL. 2001.

${ }^{5}$ Myers, K.L., Jarvis, P.A., Tyson,W.M., and Wolverton, M.J. “A Mixed-initiative Framework for Robust Plan Sketching,” In Proceedings of the 13th International Conferences on AI Planning and Scheduling, Trento, Italy, June, 2003.

${ }^{6}$ Alvarado, C. and Davis, R., "Resolving Ambiguities to Create a Natural Sketch Based Interface," In Proceedings of IJCAI2001. August 2001.

${ }^{7}$ Hsia, A., Samoylov, A., McGovern, A., and Bailey, B., "Designer's Choice: Constructing a Study to Compare Display Interfaces for Early Design Sketching," Eurographics SMB'05 - Sketch Based Interfaces and Modeling Workshop. Trinity College Dublin, Ireland. August 28-29, 2005.

${ }^{8}$ Horn, P. Autonomic computing: IBM's perspective on the state of information technology. Presented at agenda 2001, Scotsdale, Arizona, 2001, IBM T. J. Watson Laboratory, October 15, 2001.

${ }^{9}$ Murch, R. Autonomic Computing. IBM Press, 2004.

${ }^{10}$ M. Greenberg, A. Roberts, G. Edwards, P. Barros and S. Fierro. InCOA: Human-Machine-Human Collaboration in SketchBased Mission Planning. In: 2009 International Symposium on Collaborative Technologies and Systems (CTS 2009).

${ }^{11}$ Kitano, H., Asada, M., Kuniyoshi, Y., Noda, I., and Osawa, E. 1997. RoboCup: The Robot World Cup Initiative. In Proceedings of the First international Conference on Autonomous Agents (Marina del Rey, California, United States, February 05 - 08, 1997). AGENTS '97. ACM, New York, NY, 340-347. 
${ }^{12}$ Kitano, H., Asada, M., Kuniyoshi, Y., Noda, I., Osawa, E., and Matsubara, H. 1998. RoboCup: A Challenge Problem for AI and Robotics. In Robocup-97: Robot Soccer World Cup I H. Kitano, Ed. Lecture Notes In Computer Science, vol. 1395. Springer-Verlag, London, 1-19.

${ }^{13}$ Scerri P. and Ydren J. End User Specification of RoboCup Teams in "RoboCup-99: Robot Soccer World Cup III", Springer Verlag Lecture Notes in Artificial Intelligence (LNAI) 2000.

${ }^{14}$ Arkin, R.C. and Balch, T. Cooperative multiagent robotic systems. Artificial Intelligence and Mobile Robots. 277-296, 1998.

${ }^{15}$ Nourbakhsh, I.R. and Sycara, K. and Koes, M. and Yong, M. and Lewis, M. and Burion. Human-robot teaming for search and rescue. IEEE Computer Society. 72-78, 2005

${ }^{16}$ Wang, J. and Lewis, M. Human control for cooperating robot teams. Proceedings of the ACM/IEEE international conference on Human-robot interaction. 2007.

${ }^{17}$ Parasuraman, R. and Galster, S. and Squire, P. and Furukawa, H. and Miller, C. A flexible delegation-type interface enhances system performance in human supervision of multiple robots: Empirical studies with RoboFlag. IEEE Transactions on Systems, Man, and Cybernetics, Part A: Systems and Humans. 35(4), 481-493, 2005.

${ }^{18}$ Squire, P. and Trafton, G. and Parasuraman, R. Human control of multiple unmanned vehicles: effects of interface type on execution and task switching times. Proceedings of the 1st ACM SIGCHI/SIGART conference on Human-robot interaction. 2006. 